\title{
An Analysis of China-CEE Cooperation from Geo- Economic Perspective: Aspects, Layers, and the System, 2012-2019
}

\author{
Ziyu Zhou ${ }^{1, *}$ \\ ${ }^{1}$ Department of Government and International Studies, Faculty of Social Sciences, Hong Kong Baptist University, \\ Kowloon Tong, Kowloon, Hong Kong \\ *Corresponding author. E-mail: 18251110@life.hkbu.edu.hk
}

\begin{abstract}
The research purpose of this paper is to discuss whether the cooperation between China and Central and Eastern European countries can be evaluated in an approach of geo-economic perspective. In order to measure the turnout, the paper quantifies the cooperation degree into the actual numbers of consensuses that were reached by China and CEE countries in annual meetings from 2012 to 2019. Thus, the research paper finds that the cooperation is organized around with the center of economic exchanges, while the pattern can be broken down in aspects, layers and the system. Significantly speaking, this research paper is useful for future strategic cooperation between China and Central and Eastern Europe, while providing a contemporary cooperation model among nation states across regions.
\end{abstract}

Keywords: China, Central and Eastern Europe, geo-economic, connectivity, economic exchange, cultural exchange, political cooperation

\section{INTRODUCTION}

The main object of this paper is to evaluate whether the cooperation between China and Central and Eastern European countries can be evaluated in an approach of geo-economic perspective. The reasons to choose such an issue are that first recently there are debates about whether the China-CEE cooperation represents for China's geopolitical interest or contemporary cosmopolitan way of development. Besides, on the cooperation itself, it faces many critics and challenges that remain under-researched. Therefore, in order to have a comprehensive overview of the relation between China and Central and Eastern Europe, the research paper chooses the China-CEE cooperation as a whole case study. Detailly speaking, the cooperation that is taken between China and the Central and Eastern European countries is under a complicated situation that on the one hand the uprising of China including its more active policies such as the launch of the OBOR causes doubts and concerns in the international society, which worth examining. While on the other hand, after 1989 the transition of Central Eastern Europe from Socialist camp to the European Union, as well as the CEE area are traditionally considered as a geopolitical playground for great powers. By studying this unique existing relation, this research paper is useful for future strategic cooperation between China and Central and Eastern Europe, while providing a contemporary cooperation model among nation states across regions. Overall, the essay will be divided into several parts. The first section presents the current explanations for China-CEE cooperation, while the second expresses the original explanation of geo-economic perspective, purposing the hierarchical cooperation model which contains aspects, layers, and the system. The next section analyses the data of cooperation numbers from the perspectives of year and aspects (cooperation in connectivity, cooperation in direct economic exchanges, cooperation in indirect economic exchanges, cooperation in people-to-people and cultural exchanges, and overall political and functional cooperation). And the fourth section discusses the original cooperation model and details. The research paper ends with a final conclusion and shares lights on future studies. 


\section{LITERATURE REVIEW}

In fact, some of the existed studies try to explain the issue of cooperation between two entities that are connected by cooperation events, especially the relations between China and Central and Eastern Europe. Góralczyk [1] argued that in a geopolitical aspect China considers Central and Eastern Europe as a strategic place where it can broaden its interests and involvement in the CEE area. This kind of consideration was declared by Wang [2] that Europe is recognized as a key point for China to express its influence over the world by promoting the China-CEE cooperation and the OBOR (One Belt One Road) project. Also, in the perspective of geopolitics, China seeks for its interest as well as a new global order based on its foreign policy such as the OBOR. For Dungse et al. [3], these initiatives including the OBOR are designed to build up a mutually beneficial system that can connect different political communities through Eurasia or even Africa, in way of "global governance". By new institutions that appear in ethics, institutions, and societies, China tends to turn from risk avoidance to seeking opportunities in the midst of risk and aims to build its own global order where it has geopolitical and geo-economics significance to have a more important role in the international system [3]. In this approach to evaluate China's evolvement in the international society, without doubt the issues of China's uprising international power and desire are emphasized. China's goals can be discussed clearer taking the international strategy into consideration. However, as the goals of "coming up with a new world order" seem to be those are ultimate or in a long term, explaining all actions for geo-political concerns may sometimes over considers China's ambition in short term, which cannot directly shows the atmosphere of a new "order designer".

While, besides the geopolitical approach, a theory of cosmopolitanism could be applied to the content in order to evaluate China's behaviours. For Mignolo [4], a cosmopolitanism way to understand issues is to consider more on how things develop and the process, rather than considering much about the final goals, which represent for the potential ambitions in the China issues. As the world is guaranteed by the globalization of the world market, cosmopolitanism recognizes the nature limits of national states, which call for borderless cooperation. Christianity and capitalist economy, which were mentioned by Kant in the 18th century, used to be the two elements in an ideal cosmopolitan world. However, as Mignolo [4] agues, in the 21st world nowadays, it is impossible to consider a monocultural cosmopolitanism that only includes colonial civilizations such as France and England, instead, a cosmopolitanism with de-colonial characteristics would be a better choice for the world to develop. From this approach, it seems that China's active engagements (mainly reflects in economic areas) through the world actually infuses new possibilities to the content of cosmopolitanism, which could be used to evaluate China's motions during its process in return. But one thing needed to be emphasized is that the good vision of all the cultures come together to develop is somehow too ideal, as China or any other countries obviously contains some of its only interest demands within the cooperation.

\section{THEORY}

This article is trying to evaluate the cooperation between China and Central Eastern European countries in a geo-economic way. The so-called term of "geoeconomic" considers more on the process in the perspective of economic changes rather than the ambitious final goals in a geopolitical way, while still considers the geographic issues which may affect the cooperation to a certain extent. To be more detail, the whole paper will judge the China-CEE cooperation in a hypothetical view that most activities are organized for economic progress, while trying to find out a kind of pattern among different type of cooperation that could prove this hypothesis. To consider the issue in a geoeconomic way, both the economic basis and how the geographical elements act within the cooperation. Possible concerns may include what kind of deals are made as well as is there exist any kind of geographical connections or deals that can promote the economic connections. If the reality turns out to be that there is a kind of situation that the economic transaction and geographic structure appear at the same time, possibly a connection within these two types of cooperation can be introduced.

\section{THE ANALYSIS OF AGREEMENTS}

In the following section, the paper will try to analyze the basic data of the main agreements that were reached during the 17+1 countries' cooperation period from 2012 to 2019. The turnout of these cooperation can be found according to an event of meeting which is held each year since 2012. On an annual basis, the meeting is held for the eighteen countries to set up their next year's main goals and agreements. The fundamental structure of this meeting is that the political leaders of these seventeen countries (prime ministers for most of the time) will join a conference in one of the $16+1$ counties' cities, discussing the possibilities of cooperation between China and CEE, as well as evaluating the outcomes of cooperation of previous years. Up to September 2020, nine meetings have been held according to the official website of Cooperation between China and Central and Eastern European Countries (http://www.china-ceec.org, set up by the Secretariat for Cooperation between China and Central and Eastern European Countries).

In order to have a better understanding of what exact progress is made during the cooperation between China and $\mathrm{CEE}$, the writer classifies the nine meetings naturally 
in a manner of timeline. The nine meetings are listed as following:

1.China's Twelve Measures for Promoting Friendly Cooperation with Central and Eastern European Countries in 2012

2.The Bucharest Guidelines for Cooperation between China and Central and Eastern European Countries in 2013

3.The Belgrade Guidelines for Cooperation between China and Central and Eastern European Countries in 2014

4.The Suzhou Guidelines for Cooperation between China and Central and Eastern European Countries in 2015

5.The Medium-Term Agenda for Cooperation between China and Central and Eastern European Countries in 2015

6.The Riga Guidelines for Cooperation between China and Central and Eastern European Countries in 2016

7.The Budapest Guidelines for Cooperation between China and Central and Eastern European Countries in 2017

8.The Sofia Guidelines for Cooperation between China and Central and Eastern European Countries in 2018

9.The Dubrovnik Guidelines for Cooperation between China and Central and Eastern European Countries in 2019.

As the goal is to evaluate the cooperation between China and CEE. Two issues should be taken clearer in order to quantize the degree of cooperation. One is the concept of consensus, and the other one is the concept of aspect.

\section{Consensuses}

First, to make the cooperation to be seen more straight forward, the writer evaluates the China-CEE cooperation by introducing an index, consensuses that are made during the nine meetings. Consensuses are statements that represent agreements, which are reached by participants during the nine meetings. Being the only actual output right after meetings, this form of information is a kind of narration that shows the aims of the participants as well as the final stage of cognitive agreements which were made. Being the intersection that taking participants' requests into consideration, consensuses are the main visible pattern of manifestation from the guidelines carried out.

However, when browsing the nine guidelines of meetings from the website of Chinese government, one more issue needed to be noticed is that the tone of the
Chinese government may be different between different consensuses. Hence generally speaking, there actual exist two types of explanation of consensuses.

Type A cognitional consensus----a kind of consensus that are reached in the form of cognition. E.g. "China and Central and Eastern European Countries announce that they will cooperate more often in the area of connectivity." (Including statements like "encouraging companies and discussing the possibilities of building a new organization" as these consensuses are only kind of cognitive agreements)

Type B achievement consensus----a kind of consensus that are reached in the form of actual achievements. E.g. "A meeting on the topic of connectivity will be held in Beijing, China in 2016." (For example, a consensus saying that "Countries will have more connection in the area of connectivity, and there will be meetings to discuss how to improve it" can be roughly classified as Type B as it in fact gives out sort of achievements.)

Aspects

The other important issue needed to be considered when it comes to the different fields that have been discussed throughout nine meetings is that the style of presenting detail topics. In the following section, commitment issues are divided into five different aspects according to the details of guidelines in each year. These five aspects are divided on purpose by writer as for them, each one focuses on a specific area that can be used to evaluate to what extent or how far the cooperation goes. The reason for the artificial division is that in reality the governmental outlines from the Chinese government website are different from year to year due to the different expression of declaration languages and the different actual meeting routines. Therefore, each year the accurate number of aspects divided by government officials are different, which results in a disorder that topics are discussed and presented during the meetings may overlap with other perspectives in the next year. While each year the China-CEE meeting will always discuss several issues in an unfixed order, the overall areas that are discussed seldom changed. To deal with this problem, the writer chooses to reorder these agreements and divides them based on their functions.

The five aspects are listed as following:

Aspect 1 cooperation in connectivity (This perspective includes specific cooperation in the area of traffic, measures that enhance the transportation, such as building train lines and promoting infrastructure construction)

Aspect 2 cooperation in direct economic exchanges (This perspective includes investment, agriculture, manufacturing, tourism, and finance, which are ways of increasing economic exchanges.) 
Aspect 3 cooperation in indirect economic exchanges (This cooperation lies more in science, technology, innovation, health, environmental protection and energy, which focus more on technology exchange or technology development as well as green development rather than direct economic exchanges.)

Aspect 4 cooperation in people-to-people and cultural exchanges (This perspective focuses much on cultural events such as summer camps or culture forum.)

Aspect 5 overall political and functional cooperation (including functional cooperation that contributes to the cooperation itself, and local communication between capitals and cities.)

Acknowledging the issues of consensuses and aspects, an evaluation can be drawn out in both ways of timeline level and aspect level. In the following section of presenting data by year, the discussion will focus more on the annual development of the cooperation scale, while in the section of aspect the cooperation will be evaluated based on the annual change of specific aspects as well as comparison between different aspects.

\subsection{Section I: Data by Year}

The following statistical graphs show the total number change of the consensuses from 2013 to 2019. Also, a discussion about the appearance of peaks and the issue of periodization will also be presented in order to have a more systematic look at the cooperation between China and CEE in the level of timeline.

As Figure 1 shows, from 2013 to 2019, the planned consensuses have a rising trend in general as in 2013 the number of consensuses was 41, while in 2019 there were 85 consensuses that have reached by China and CEE. For each year from 2013 to 2019, except year 2014 and year 2017, there has been a significant increase comparing the later year with a random former year (specially in 2016 the number equals the number in 2014). According to the graph, two peaks appears in 2014 and 2017, of which numbers are 74 and 104.

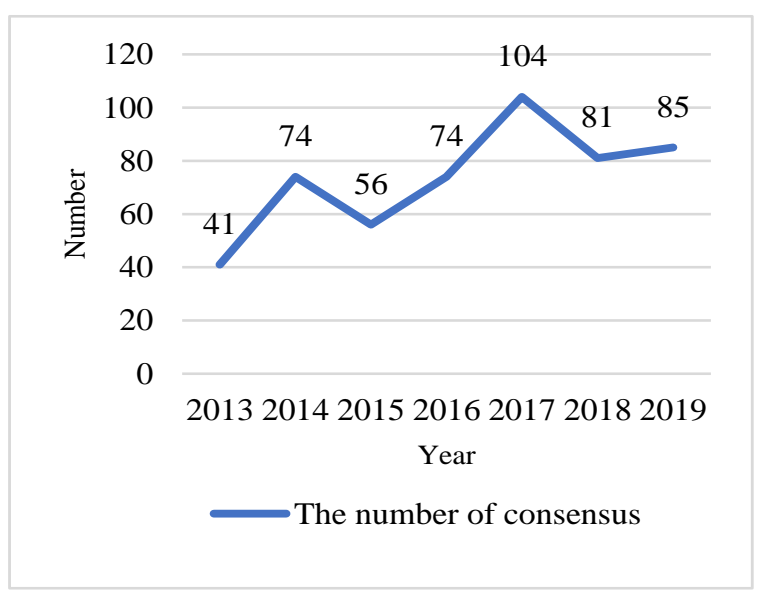

Figure 1 The number change of consensus 2013 2019

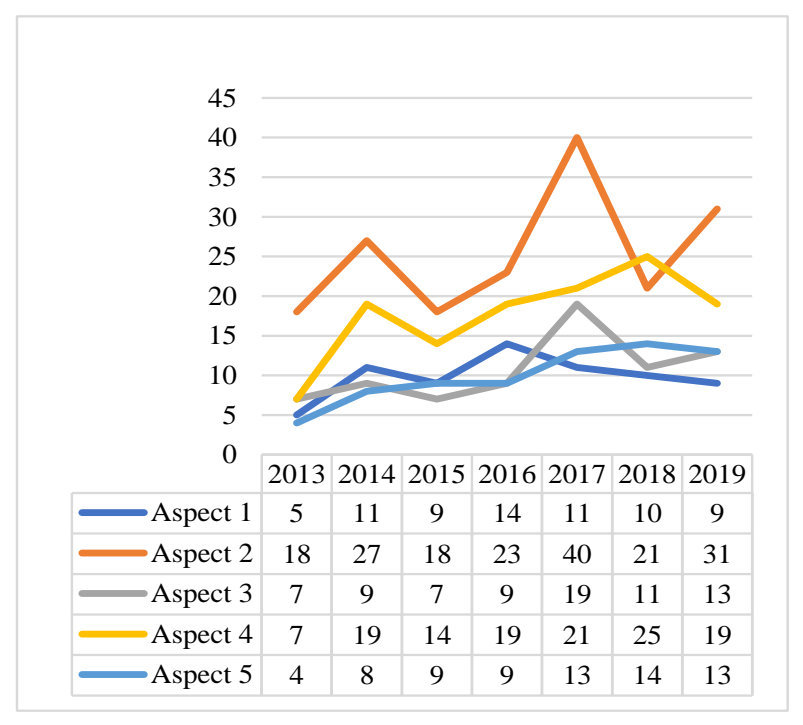

Figure 2 The number change of consensus 2013 2019 (Aspects)

Though the number change of consensuses from 2013 to 2019 has a gradually rising trend, the appearance of the two peaks in 2014 and 2017 needs further discussion. The occurrence of two highest pecks can be explained according to the graph figure 2 The Number Change of Consensus 2013 2019 (Aspects), which compares the five aspects year by year from 2013 to 2019. It can be found that from 2013 to 2019, the numbers of Aspect 2 (economic cooperation) maintained in a high level (higher than any other aspects in any year, except in 2018, in which the number of Aspect 4 was more than that of Aspect 2). Meanwhile, compared with other numbers within the Aspect 2, numbers in both 2014 and 2017 were also extremely high than any other numbers of Aspect 2 from 2013 to 2019. Therefore, there are enough evidence to prove that the appearance of two peaks in 2014 and in 2017 is due to the high increase in the area of economic cooperation while numbers in other aspects remained moderate.

\section{Periodization}

Based on the above findings, the cooperation between China and CEE up to 2019 can be periodized into two periods of time. The first period is from 2013 to 2016 , which is a stage of start-up. In this stage the cooperation between China and CEE had a moderate quantity of consensuses that the numbers of years in the period of 2013 to 2016 are all less than or equal to 74 , the number of consensuses in 2016. The second period of time for the China-CEE cooperation is from 2016 to 2019. It is a period of development. During this period, the number level of this period was equal or greater than the number of periodization point (74), or even reached the other peak (104) in 2017. Meanwhile, the two peaks are also divided by the first and second period that in each period the development of the number of consensuses the two 
peaks are placed in the middle period, and both periods experience a trend of considerable rising and slightly reduction.

The reason or evidence that support dividing the timeline of the cooperation into these two periods is that in 2015, apart from The Suzhou Guidelines for Cooperation between China and Central and Eastern European Countries, a mid-term cooperation plan, which is The Medium-Term Agenda for Cooperation between China and Central and Eastern European Countries, was carried out in order to consolidate and strengthen the cooperation relations between China and CEE. While the 12 measures signed in 2012 built a chance for China and $\mathrm{CEE}$ to begin their sizable cooperation, the mid-term agenda offered the cooperation system a direction for further development. In fact, China's Twelve Measures for Promoting Friendly Cooperation with Central and Eastern European Countries in 2012 and The MediumTerm Agenda for Cooperation between China and Central and Eastern European Countries are two special meetings among the nine. The former one works as a starting plan emphasizing the beginning of cooperation between China and CEE, while the later one is a mediumterm plan emphasizing that the cooperation had been achieved to a certain degree, and the overall plan was needed to be widened and deepened. Therefore, before and after the proclamation in 2015 there were differences in cooperation scope, which was reflected in the growing data in the graphs above.

In details (as shown in Figure 3 and Figure 4), according to the official website, the total number of overall measures in 2012 was 17, while the highest number appears in Aspect 2 and Aspect 4. However, the total number of overall measures went up to 78 during the medium-term meeting in 2015 (nearly 459\% compared with the total number in 2012). The highest number from Aspect 1 to Aspect 5 appears in Aspect 2, which has 26 measures in the area of economic exchange.

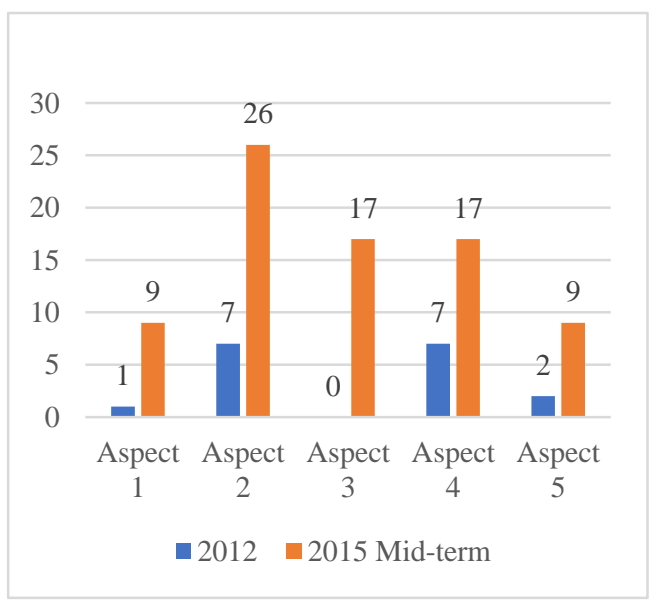

Figure 3 The number change of overall measures in 2012 \& 2015 Mid-term

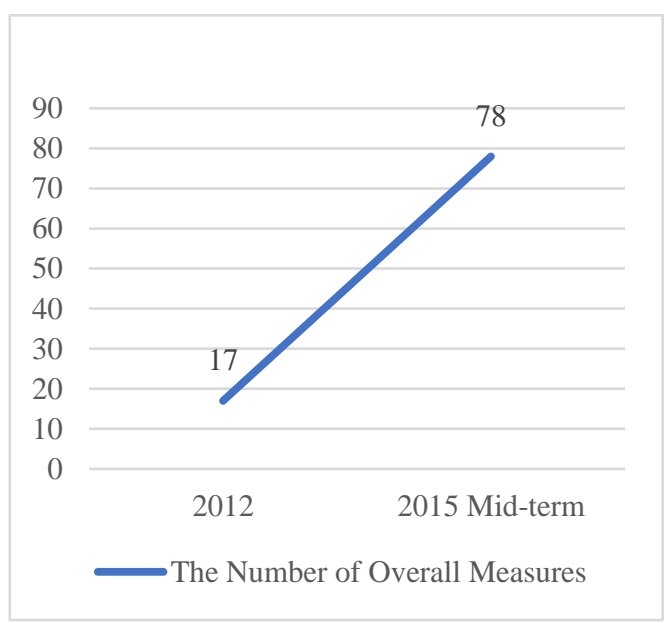

Figure 4 The number change of overall measures

\subsection{Section II: Data by Aspects}

In this section, the analysis will discuss the issue of data from the way of different aspects. As mentioned previously, there are five divided aspects that are taken into consideration: connectivity, direct economic exchanges, indirect economic exchanges, people/culture exchanges, and overall political and functional cooperation. Generally speaking, though it is hard to tell which aspect takes more significance than the others, from an amount counting level, all the aspects can be put into a three layers inverted triangle-shape model, which indicates the different weights among different aspects (see Figure 5) according to the consensuses. From 2012 to 2019, the China-CEE cooperation put the largest effort in economic and culture exchanges, following which is the aspect of political and functional consensuses, and the technology cooperation that leads to indirect economic exchanges being the larger effective one. And the last part that takes the least percentages is the connectivity cooperation (see Figure 6). As the cooperation devotes huge effort in social economic exchanges, the cooperating model is constructed based on an economic root, using institutional consensuses to build structural system. And it promotes the above consensuses through the infrastructure construction as carriers. In the rest part of this section, the paper will first present the detail data of different aspects, and then analyze the consequences based on specific aspects separately with examples. Besides, further discussions about the relations between different layers of aspects (the Core-Structural-Carrier model, as shown in Figure 5) will be presented. Finally, issues of how to qualitative define the China-CEE cooperation and why the reality turnout to be like the present will be elaborated with emphasis, considering both the characteristics of layers and specific aspects. 


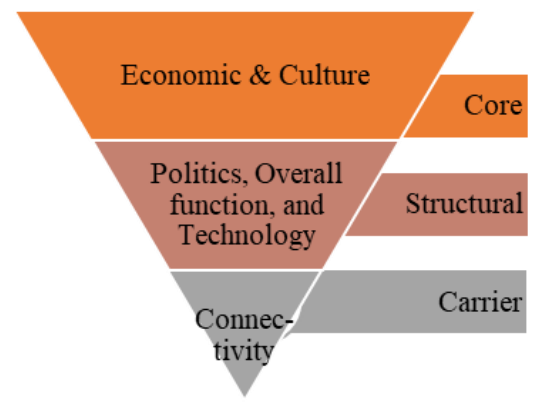

Figure 5 The geo-economics system

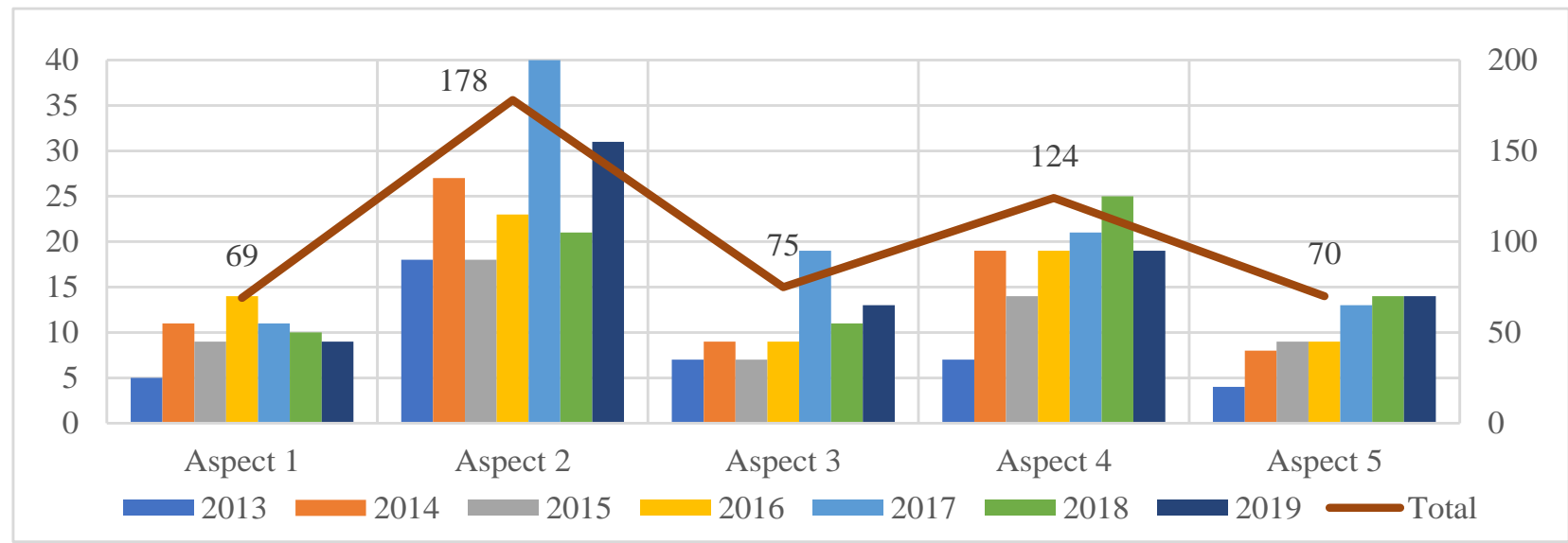

Figure 6 An overall review From Aspect 1 to Aspect 5 (2013 2019)

\section{Aspect 1. Cooperation in connectivity}

The Aspect 1 mainly focuses on infrastructure construction events. For example, building up railways and highways. Promoting harbor and setting up customs cooperation can also be one of the parts of connectivity. For the cooperation in connectivity, the main finding is that the consensuses made from 2013 to 2019 mainly appear in small numbers that in the seven-year time there was no year above the number of 14 in 2016, and the smallest number is 5 in 2013, while the total number is 70 , which is the smallest among the five aspects. For the comparison between two types of consensuses, it can be found that there are more Type A consensuses than Type B for most of the years but not too many. Therefore, the cooperating modality of connectivity contains both small numbers of cognitive agreements and real cooperation, while in detail the real cooperation is small in number, but discourse agreements sometimes are easier to make.

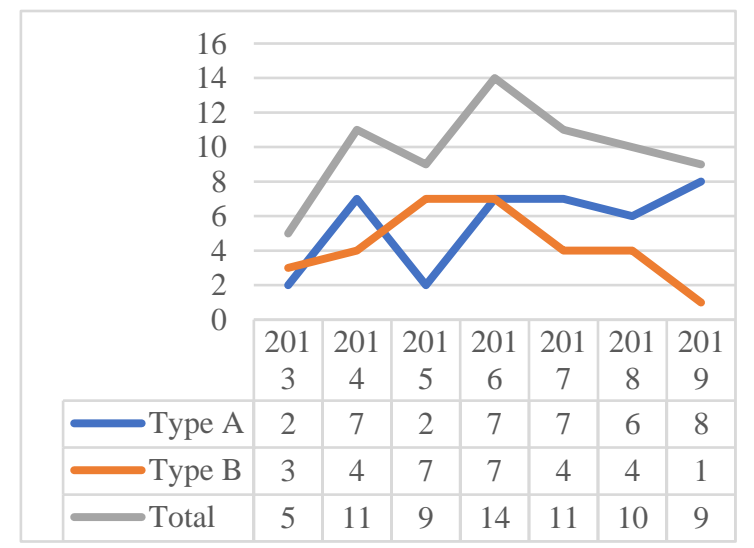

Figure 7 The Number Change of Consensus in Aspect 1

\section{Aspect 2. Cooperation in direct economic exchanges}

The cooperation in the Aspect 2 is one of the majorities among the five aspects. Those direct economic exchanges include all kinds of economic actions that directly create value, such as direct investment, primary, secondary and tertiary industries promotion, and finance. From the outlines it can be found that this type of cooperation in fact is the biggest form of cooperation that it has reached the number of 178 in five years, with the highest peak-number of 40 in 2017 and the highest bottom-number of 18 in 2013. For the issue of Type A and Type B, the major part of Aspect 2 is made up by Type A, which indicates that the main implementation 
way of this aspect is always an actual event for investment, trade, or economic institution, rather than some cognitive agreements.

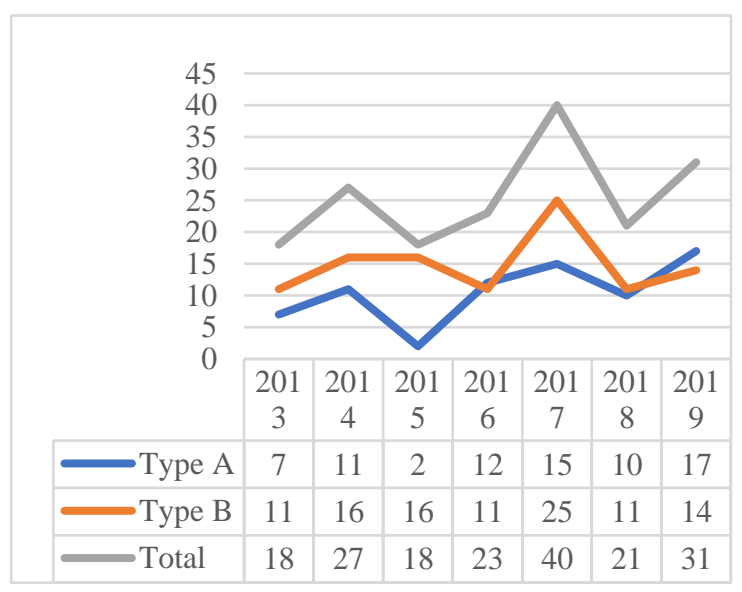

Figure 8 The Number Change of Consensus in Aspect 2

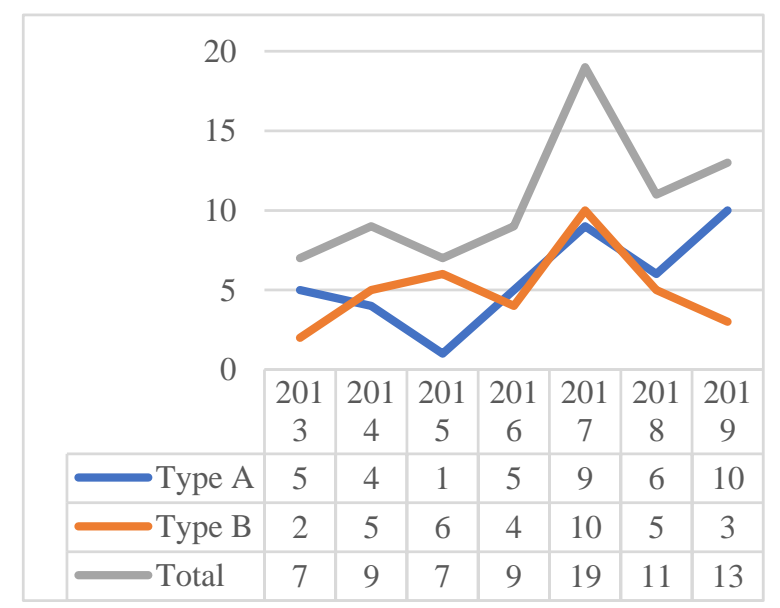

Figure 9 The Number Change of Consensus in Aspect 3

\section{Aspect 3. Cooperation in indirect economic exchanges}

The Aspect 3 reflects the agreements about indirect economic exchanges, which may not lead to direct economic exchanges. Instead, those areas of production indirectly affect the productivity and economic status. Though this form of cooperation in science and technology, as well as environmental innovation is not the biggest, nor it is the least. From 2013 to 2019, there are 75 consensuses appeared in the Aspect 3 in total, with the peak number of 19 in 2018 and the bottom number of 7 in 2013. On the level of Type, before 2016 there were more actual cooperation than cognitions, while after 2016 the actual cooperation reduced, and cognitive consensuses took more percentages. It can be seen that the difference between Type A and Type B is not too huge in seven years of time.

\section{Aspect 4. Dynamic people-to-people and cultural exchanges and cooperation}

The cooperation based on linking people and people from other parts of the World mainly show up as cultural festivals to some degree. This kind of actions sometimes creates economic benefits but with more importance for culture exchange means. For the outlines, this aspect is the other main form of cooperation besides the Aspect 2. From 2013 to 2019, the total number of Aspect 4 remained in a high level that most of the numbers are above 14, with the highest number 25 in 2018, and the bottom number 7 in 2013. The pattern in Type of the Aspect 4 is obvious that there were always more Type B consensuses than Type A except in the year 2019, and the gap was distinctive. It turned out to be that there are large number of actual events held on behave of culture exchange in the relationship between China and the Central-Eastern Europe, while the cognitive agreements were less but in a trend of rising in the long term.

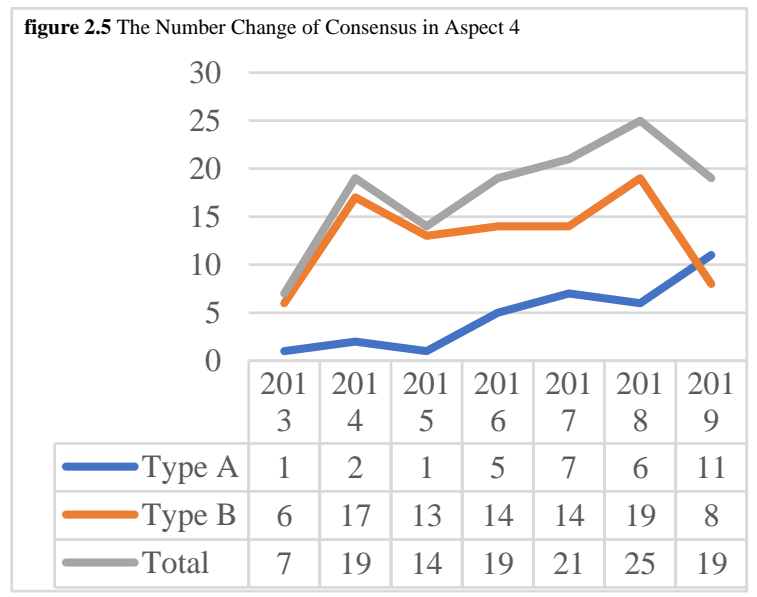

Figure 10 The Number Change of Consensus in Aspect 4

Aspect 5. Overall political and functional cooperation

The majority of the Aspect 5 from the outlines are the functional designs, which aim at providing the ChinaCEE a better platform to communicate and to practice any other aspects. From 2013 to 2019, the cooperation in overall political and functional cooperation was in a trend of rising, from the bottom number of 4 in 2013 to the peak number of 14 in 2018. Instead of Type A consensuses that aim at offering cognitional ideas, Aspect 5 has more Type B consensuses that set up actual system to promote the China-CEE cooperation (except year 2019). 


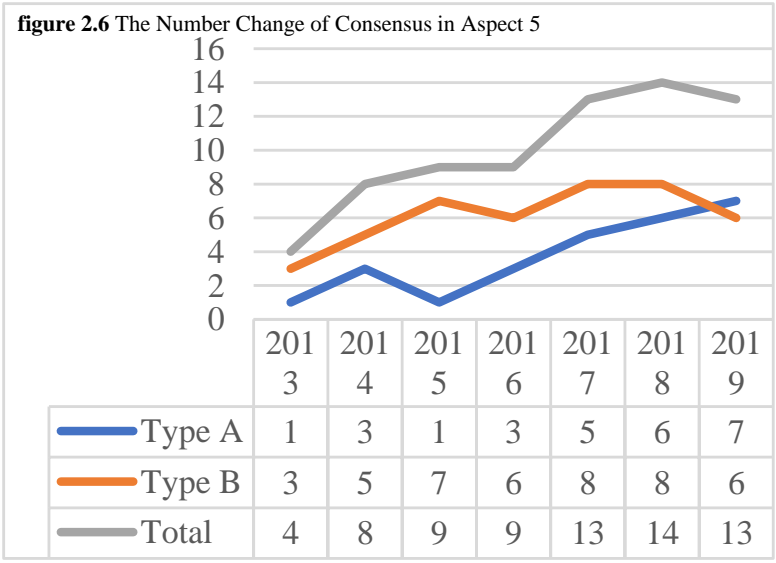

Figure 11 The Number Change of Consensus in Aspect 5

\section{DISCUSSIONS: ASPECTS, LAYERS, AND THE SYSTEM}

This part mainly focuses on explaining why there exist differences between aspects that have different numbers of consensuses, as well as trying to draw out a formulative model (the Core-Structural-Carrier Model. See Figure 5) that could explain the relationship and the connections between different groups of aspects (deliberately generalized by the writer) based on the concept of geo-economics.

\section{Aspects}

First, for the part of explaining why the aspects turned out to be in this way. One of the biggest possible reasons is that it is the inherent characteristics of different aspects (or even the differences of layers that each layer of aspects has own characteristics) cause the discrepancy. Those characteristics can be found from all the perspectives of how an activity is constructed, all the way to how it could be put into practice, the problem drew out within the practicing, as well as how it should be ended with specific benefits. The following paragraphs take two of the typical cases from the three layers as examples: one is the bottom layer, the issue of connectivity, and the other one is peak layer, the issue of cultural exchange.

For the issue of connectivity, as this type of consensuses focuses large on infrastructure construction like roads and harbours, it takes governments years to negotiate on the problems of construction plans, as well as the following evaluating concerns including the budget and the internal law compliance. Among the cooperation between China and the CEE, one of the most important projects that is under promoting is the Budapest-Belgrade Railway, which has been on plan (The Bucharest Guidelines for Cooperation between China and Central and Eastern European Countries) since November 2013. This $350 \mathrm{~km}$ railway is scheduled to be an important issue that indicates the great cooperation between China and two of the CEE countries, Serbia and Hungary. However, according to Brînză [5], the railway construction was still not finished in 2020, seven years after the agreement was reached. What's more, during the seven years of time, there existed large scales of negotiations between different shareholders due to several different self-interest concerns such as prospective earnings, the huge loan issues, and the tending legality suspicion from the EU [5]. More newly coming issues tended to appear during the constructing process. Therefore, it can be found that in the perspective of connectivity, even one fruitful achievement is needing for years of cooperation and long-time commitments of resources in quantity, in order to keep the project on plan. So, due to this nature of connectivity issues, the consensuses on connectivity take the least among all the outlines from 2013 to 2019.

Unlike connectivity taking the least, on the other side of the inverted triangle, the cultural exchange cooperation has the second largest number of consensuses among all outlines and occupies the top layer of the triangle together with the economic cooperation. But just like the aspect of connectivity, it is also the peculiar characteristics of cultural exchanges that led to the great deal of cooperating projects. Compared with the connectivity issues, the difficulties to realize cultural exchanges are much easier. For the consensuses, activities of cultural exchanges are often introduced and carried out by the means of events. From an official level there are seminars to discuss culture issues, as well as in a folk level there are plenty of festivals and summer/winter camps. These activities do not require for long-time design or huge investment. Instead, short-term human resources transference with enough funds make it possible for any members of the cooperating partner country to hold a cultural meeting. According to a 2016 news reported by the People's Daily from China [6], there were nearly 40 cultural events that were related to the China-CEE cooperation within 2016. Setting up new cultural projects is also much easier and acceptable by different shareholders as this kind of activities does not too much power relinquishment and interest balance. Besides, beneficial from the short execution cycle, cultural exchange events can be put into practice within a short time and can be promoted annually, which extensively enriched the number calculation each year. Therefore, this aspect with mass number appeared due to the nature that it has higher accessibility and achievability, which make it easy to be coordinated with.

\section{Layers}

As mentioned in the previous paragraphs, the relations between different aspects, which further derived into the relationships between different layers is the issue with great essentiality in the discussion part. In order to have a better understanding of how these three different layers of aspects connect with each other, how the intrinsic essence of the China-CEE cooperation exists should be defined. For the writer, the cooperation is built 
based on the concept of geo-economic. Wigell [7] considered "geo-economics as the geostrategic use of economic power and constructed two typologies: strategic frame (competitive or cooperative) and economic power (as goal or means)". In other words, economic events can be taken great importance as a starting perspective to evaluate an issue that contains much economic elements like trade and economic negotiations. Obviously, for the China-CEE relations, more cooperation strategic frames are constructed as between these two economic entities, more cooperation issues were made and there are less competitive issues. But how to analyze the connections within the geoeconomic cooperation relationship? Take the ChinaASEAN cooperation issue as an example, according to the research done by Wang et al. [8], embracing the core mechanism of geo-economics, different perspectives have special functions to promote the geo-economics. To be brief, Wang et al. [8] argued that the geo-economics are driven by economic drivers, political drivers, cultural drivers, and location drivers. The economic drivers enhance geo-economics by the benefit-sharing mechanisms such as market, policy, and technical institutions. And the political drivers offer a set of institutional mechanisms like cooperation, negotiation, and coordination, while for the cultural drivers and the location drivers, a kind of transmission mechanism and selection mechanism are provided to evaluate the consequence of driven forces for geo-economic relationships [8].

Based on the above consideration, the analysis dimension from Wang et al. [8]'s study that considers the geo-economic issues by different aspects is acceptable and easy to understand. Dividing the whole cooperation into several parts that all have the core of geo-economic makes the cooperation distinctive to evaluate. Thus, any event within this geo-economic system can be seen as an action to promote the geo-economic issues with its own characteristics. Meanwhile, as the structure of EconomicPolitical-Cultural-Location highlights the inherent attributions of these perspectives, the own characteristics can be fully observed when considering the perspectives. Therefore, following this way of evaluation, the connection and the different unique functions can be both involved.

However, a further perspective can be emphasized and be introduced to deepen the relationship between those existed aspects, which is the aspect of connectivity. As the events of building up roads and harbours sometime can represent for both economic and political meanings, the connectivity perspective can be separately defined as a driver that crosses the existed aspects and gives them supports to carry out. Besides, the issue of connectivity brings solutions for the geographic problems for geo-economic cooperation, as economic and political events that are put into practice are built following the existed geographical locations, and the connectivity issues can improve it, as well as it can shorten the haulage time. Therefore, its importance should be strengthened that for geo-economic cooperation, how different entities are connected with each other should be taken into equal consideration.

Back to the China-CEE relationships the object. Based on the above discussions, three layers of aspects are introduced, which including the existed economic, political, and cultural parts, with the connectivity part at the bottom. On a quality level, all the three layers are equal that any of the three has its own influence on the geo-economic cooperation in totally different ways, while the difference in shape areas represent for the frequency of occurrence. The Layer of Economic \& Culture which with the biggest number represent for the core status that the economic issues are the largest quantity within the geo-economic approach. The Layer of Political, Overall function, and Technology represents for the political means that are taken to promote institutional mechanism. And the last but not the least, the Layer of Connectivity works as the least in number part to be the bottom part of the geo-economic triangle that it strengthens the importance of the geographic issues and objective supports.

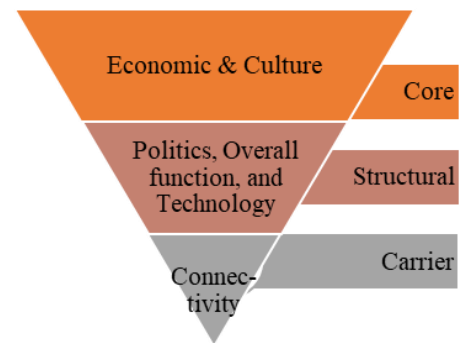

Figure 12 The geo-economics system

System

Following the concept of layers, the three layers of aspects in fact can be seen as a whole subjective body within which layers nest with each other and construct a systematical structure that overall support the cooperation to be promoted through years. In details, the core of the geo-economic cooperation, which is the layer with the largest number (economic and cultural cooperation), makes up of the most important main body of the system. As this mechanism decides what benefit could be gained from the cooperation, it largely decides the extent the cooperation can go further. And, based on these core values, a series of structural mechanisms are created by the shareholders (China and CEE countries) to support the cooperation in order to achieve the prospective benefits. This layer includes those political and functional support that can indirectly support the economic growth. Though this layer of aspects all focuses more on functional issues, they are still associated with geoeconomics as they are institutional mechanisms aim at gaining higher economic cooperation. The last Layer of Connectivity works in the carrier role among the system, 
which offer the essential productive factors more possibilities to move faster within the cooperation period.

That is to say, the relationship between the three layers within the system of China-CEE cooperation is in fact assumed to be a dynamic balance. Within the system the total number-calculation among three layers is decreased from top to bottom (Core-Structural-Carrier), while the unit cost among the three layers is decreased from bottom to top (Carrier-Structural-Core). This phenomenon is due to the characteristics of aspects and the layer role in the system, which has been discussed in former paragraphs. Also, the system is a dynamic loop operation. The huge resources and funds required by the core layer of Economic \& Culture travel through the cooperating areas by the transportation of connectivity constructions, and these objective entities are ensured and protected by the structural functions, the policy and technology support. During the journey, if the system is operated well, the cooperation is supposed to have great progress in geo-economic issues, which further prove the benefits and the necessity of the China-CEE cooperation. And this positive expectation logically reflects in the enrichment of economic resources in a geo-economic perspective. More resources argue for flexible structural supports as well as more capable carrier supports. Therefore, if the system to achieve the geo-economic goals is supposed to work regularly with efficiency, none of the three layers can be left behind.

\section{CONCLUSION}

In Summary, the research first described the existed studies on the situation of the China-CEE cooperation. Then it tried to explain the main characteristics of the issue of geo-economic in order to have a better understanding of the consequence evaluating outlines and purposed a hypothetical model (aspects, layers, and the system). After that the paper divided the cooperation outlines detailly in year perspectives and five aspects, which quantitated the cooperation so that a distinctive result can be drew out. Following that the essay tried to verify the former hypothesis, which was based on the geo-economic concept, and developed the details of the possible cooperation model (aspects, layers, system) of the China-CEE relation. Though the research paper tried to have an overall glance at the Chin-CEE relation, it has several limitations. First, the way of measurement could be more developed and contain more cases for real activities and cooperation, as the paper only evaluate the documents of cooperation. Besides, this case study offered detail account of China-CEE cooperation, but the cooperation between different areas within the ChinaCEE cooperation might be different based on their own context and reality. So possibly the detail cooperation between countries cannot suitably follow the discuss pattern of geo-economy. Nevertheless, the significance of the essay can be revealed in two ways. Firstly, the case study provided a glance at the current situation of the
China-CEE cooperation that the past development is clearer, and on a strategic level, more targeted researches based on geo-economic approach on the China-CEE relation can be carried out, which may also be helpful for future strategic design. Secondly, the geo-economic model of aspects-layers-the system can be seen an analysis model that can be applied to the cooperation evaluation among other nation states across regions in a geo-economic approach. Following this way, future studies could develop a new measure of degree of evaluation, not only include consensuses but other perspectives of cooperation. Besides, the geo-economic model could be also applied to other cases that may bring some new findings.

\section{REFERENCES}

[1] B. Góralczyk, China's interests in central and eastern Europe: enter the dragon, European View. 16(1) (2017) 153-162. DOI: https://doi.org/10.1007/s12290-017-0427-9

[2] Y. Wang, The Belt and Road Initiative. What will China offer the world in its rise, New World Press, Beijing, 2016.

[3] Y. Dungse, F. Chidozie, O.O. Aje, Oscillating between the great powers and China's quest for a new world order, Acta Universitatis Danubius. 11(2) (2018) 194-210.

[4] W. D. Mignolo. De-colonial cosmopolitanism and dialogues among civilizations, in: G. Delanty (Eds.) Routledge Handbook of Cosmopolitanism Studies, Routledge Handbooks Online, 2012, pp. 85-100.

[5] A. Brînză, China and the Budapest-Belgrade railway saga, Web News, The Diplomat, 2020.

[6] M. Peng. China, CEE to celebrate cultural exchange year with nearly 40 events, Web News, Global Times, 2016.

[7] M. Wigell, Conceptualizing regional powers' geoeconomic strategies: neo-imperialism, neomercantilism, hegemony, and liberal institutionalism, in: U. Volz, W. Shen (Eds.), Asia Europe Journal, vol. 14, Springer, 2016, pp. 135 151. DOI: https://doi.org/10.1007/s10308-015-0442$\mathrm{X}$

[8] S. Wang, X. Xue, A. Zhu, Y. Ge. The key driving forces for geo-economic relationships between China and ASEAN countries, Sustainability. 9(12) (2017) 2363.

DOI: 